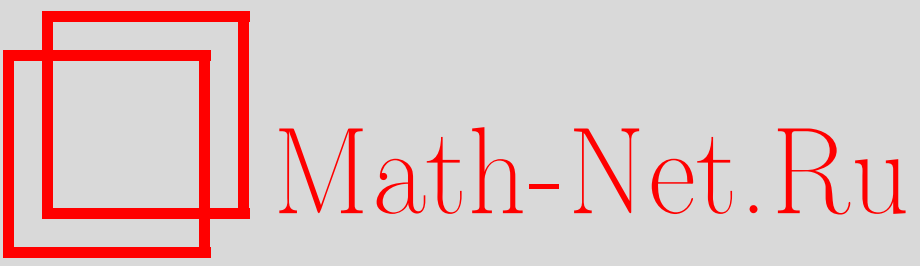

С. М. Сергеев, Оператор эволюции для некоторого квантового маятника, ТMФ, 2004, том 138, номер 1, 35-40

DOI: https://doi.org/10.4213/tmf14

Использование Общероссийского математического портала Math-Net.Ru подразумевает, что вы прочитали и согласны с пользовательским соглашением

http://www.mathnet.ru/rus/agreement

Параметры загрузки:

IP : 52.87 .193 .239

26 апреля 2023 г., 11:50:00 


\section{ОПЕРАТОР ЭВОЛЮЦИИ ДЛЯ НЕКОТОРОГО КВАНТОВОГО МАЯТНИКА}

Предложено соотношение нового типа для рядов по простой алгебре Вейля. Это соотношение позволяет выразить, в частности, оператор эволюции некоторого квантового маятника через его гамильтониан.

Ключевые слова: простая алгебра Вейля, компактный квантовый дилогарифм.

Компактный $q$-дилогарифм ( $q$-экспонента) по определению [1] есть

$$
(x ; q)_{\infty}=\prod_{n=0}^{\infty}\left(1-q^{n} x\right)=\sum_{n=0}^{\infty} \frac{(-1)^{n} q^{n(n-1) / 2}}{(q ; q)_{n}} x^{n}
$$

где предполагается $|q|<1$ и $(x ; q)_{n}=(1-x)(1-q x) \ldots\left(1-q^{n-1} x\right)$. Если аргументы $q$-дилогарифма принадлежат простой алгебре Вейля

$$
\mathbf{u v}=q \mathbf{v u}
$$

то $q$-дилогарифм используется для построения группы рациональных преобразований (2). Например, операторнозначная функция $(\mathbf{u} ; q)_{\infty}$ совершает преобразование

$$
(\mathbf{u} ; q)_{\infty} \mathbf{v}(\mathbf{u} ; q)_{\infty}^{-1}=\mathbf{v}(1-\mathbf{u})^{-1}
$$

Различные замечательные соотношения для $q$-рядов на простой алгебре Вейля (2) могут быть применены в квантовой механике [1]. Наиболее известными являются теорема сложения и пентагональное тождество:

$$
(\mathbf{u} ; q)_{\infty}(\mathbf{v} ; q)_{\infty}=(\mathbf{u}+\mathbf{v} ; q)_{\infty}, \quad(\mathbf{v} ; q)_{\infty}(\mathbf{u} ; q)_{\infty}=(\mathbf{u}-\mathbf{v u}+\mathbf{v} ; q)_{\infty}
$$

В данной работе представлен более сложный результат, касающийся следуюшей функции, состоящей из пяти сомножителей:

$$
\mathbf{s}=\left(a_{1} \mathbf{v}^{-1} ; q\right)_{\infty}\left(b_{0} \mathbf{u} ; q\right)_{\infty}\left(-c_{0} \mathbf{v} \mathbf{u} ; q\right)_{\infty}\left(a_{0} \mathbf{v} ; q\right)_{\infty}\left(b_{1} \mathbf{u}^{-1} ; q\right)_{\infty}
$$

* Лаборатория теоретической физики им. Н. Н. Боголюбова, Объединенный институт ядерных исследований, Дубна, Московская обл., Россия; Max-Planck-Institut für Mathematik, Bonn, Germany. E-mail: sergeev@thsun1.jinr.ru; sergeev@mpim-bonn.mpg.de 
где $\mathbf{u}, \mathbf{v}-$ генераторы Вейля и $a_{0}, a_{1}, b_{0}, b_{1}, c_{0} \in \mathbb{C}$. Пусть

$$
\mathbf{h}=H(\mathbf{u}, \mathbf{v})=a_{0} \mathbf{v}+b_{0} \mathbf{u}+a_{1} \mathbf{v}^{-1}+b_{1} \mathbf{u}^{-1}-c_{0} \mathbf{v} \mathbf{u}-c_{1} \mathbf{v}^{-1} \mathbf{u}^{-1},
$$

где $c_{1}=a_{1} b_{1}$. Полином $\mathbf{h}$ играет роль гамильтониана $\mathbf{s}$ в следуюшем смысле. Оператор $\mathbf{s}$ определяет рациональное преобразование

$$
\mathbf{u} \mapsto \mathbf{u}^{\prime}=\mathbf{s u s}^{-1}, \quad \mathbf{v} \mapsto \mathbf{v}^{\prime}=\mathbf{s v s}^{-1},
$$

где в результате преобразования (3) $\mathbf{u}^{\prime}$, например, принимает вид

$$
\mathbf{u}^{\prime}=\tilde{\mathbf{u}}-\mathbf{v}\left(a_{0}-c_{0} \tilde{\mathbf{u}}\right) \tilde{\mathbf{u}}\left(1-b_{0} \tilde{\mathbf{u}}\right)^{-1}, \quad \tilde{\mathbf{u}}=\mathbf{u}\left(1-a_{1} \mathbf{v}^{-1}\right)^{-1} .
$$

Можно легко проверить, что $H\left(\mathbf{u}^{\prime}, \mathbf{v}^{\prime}\right)=H(\mathbf{u}, \mathbf{v})$, т.е. $\mathbf{h}$ и в самом деле является инвариантом $\mathbf{s}$,

$$
\mathbf{s h}=\mathbf{h s} .
$$

Полином (6) иногда называется гамильтонианом своего рода квантового маятника, хоть форма эволюционного отображения несколько необычна.

Оператор $\mathbf{s}$ и его инвариант $\mathbf{h}$ возникали в работе [2] как оператор эволюции и его гамильтониан для простейшей ситуации в некоторой иерархии $(2+1)$-мерных интегрируемых систем. В работе [2] была сформулирована следуюшая

Гипоте3А 1. Операторнозначная функиия $\mathbf{s}$, определенная формулой(5) как ряд no $\mathbf{u}, \mathbf{v}$

$$
\mathbf{s}=\sum_{n, m \in \mathbb{Z}} f_{n, m}\left(a_{0}, a_{1}, b_{0}, b_{1}, c_{0}\right) \mathbf{v}^{n} \mathbf{u}^{m}
$$

может быть переписана тождественно как $\mathbf{v}^{n} \mathbf{u}^{m}$-разложсение

$$
\mathbf{s}=\sum_{k=0}^{\infty} g_{k}\left(a_{0} a_{1}, b_{0} b_{1}, c_{0} a_{1} b_{1}\right) \mathbf{h}^{k}
$$

для произвольных $a_{0}, a_{1}, b_{0}, b_{1}, c_{0}$.

Чтобы получить (10) из (11), нужно совершить бесконечное частичное суммирование: пусть

$$
\mathbf{h}^{k}=\sum_{n, m=-k}^{n, m=k} \chi_{k, n, m} \mathbf{v}^{n} \mathbf{u}^{m}
$$

тогда утверждение гипотезы 1 состоит в том, что ряды

$$
f_{n, m}=\sum_{k=0}^{\infty} g_{k} \chi_{k, n, m}
$$

являются сходящимися. Заметим, что тождества (4), будучи частными случаями представления $\mathbf{s}$ в виде функший соответствуюшего $\mathbf{h}$, содержат конечные частичные суммирования типа (13). 
Далее, мы можем предположить форму функции (11). Обозначим для краткости

$$
a=a_{0} a_{1}, \quad b=b_{0} b_{1}, \quad c=c_{0} a_{1} b_{1} .
$$

Пусть для произвольных комплексных $h, a, b, c$

$$
S(x)=\left(\begin{array}{cc}
1-h x+b x^{2} & 1 \\
q^{-1} x(1-x)\left(a-q^{-1} c x\right) & 0
\end{array}\right)
$$

$$
S_{n}(x)=S(x) \cdot S(q x) \cdot S\left(q^{2} x\right) \cdots S\left(q^{n-1} x\right) .
$$

Для $|q|<1$ можно легко убедиться в том, что

$$
S_{\infty}(x)=\left(\begin{array}{cc}
s(x) & s(x) \\
\tilde{s}(x) & \tilde{s}(x)
\end{array}\right), \quad \tilde{s}(x)=q^{-1} x(1-x)\left(a-q^{-1} c x\right) s(q x) .
$$

Согласно этому определению $s(x)$ является также функцией $a, b, c$ и $h$ :

$$
s(x)=s(a, b, c ; h ; x), \quad s(a, b, c ; h ; 1)=s(b, a, c ; h ; 1) .
$$

Второе равенство представляет собой примечательную симметрию, хорошо скрытую в определении (17) функции $s$. В эквивалентной формулировке, если $F(x)$ - решение функционального уравнения

$$
F(x)=1-h x+b x^{2}+\frac{x(1-q x)(a-c x)}{F(q x)}
$$

при $F(0)=1$, получаем $s(x)=\prod_{n=0}^{\infty} F\left(q^{n} x\right)$.

ГипотеЗА 2. Oператор $\mathbf{s}$ как функция $a, b, c u \mathbf{h}$ есть не что иное, как

$$
\mathbf{s}=s(a, b, c ; \mathbf{h} ; 1) \text {. }
$$

Для функции s, данной формулой (20), мы проверили предполагаемое равенство (13) аналитически как ряд по $q$ до $q^{21}$.

Происхождение формулы (20) может быть пояснено следующим образом.

Классический предел. Рассмотрим сначала ситуацию, когда $q=1$, так что $\mathbf{u}, \mathbf{v}, \mathbf{h}=u, v, h \in \mathbb{C}$, где $h=H(u, v)$ дан формулой (6) с коммутативными $u, v$. Как хорошо известно [3], в этом случае вместо соотношения алгебры Вейля (2) имеет место классическая алгебра Пуассона $\{u, v\}=u v$.

Уравнения движения (например, для $u$ ) принимают вид

$$
\frac{d u}{d t} \equiv \dot{u}=\{h, u\}
$$

что может быть переписано после исключения $v$ в виде

$$
\dot{u}^{2}=u^{2}\left(h-b_{0} u-b_{1} u^{-1}\right)^{2}-4 u^{2}\left(a_{0}-c_{0} u\right)\left(a_{1}-c_{1} u^{-1}\right) .
$$


Конечно же, правая часть уравнения (22) является дискриминантом уравнения $H(u, v)=h$ по $v$. Функция $v$ в терминах $u$ и $\dot{u}$ имеет вид

$$
v=\frac{h-b_{0} u-b_{1} u^{-1}-\dot{u} u^{-1}}{2\left(a_{0}-c_{0} u\right)} .
$$

Преобразование $u, v \mapsto u^{\prime}, v^{\prime}(7)$ эквивалентно сдвигу ${ }^{1)} t \mapsto t+\tau$, где

$$
\tau=\int_{u}^{u^{\prime}} \frac{d u}{\dot{u}}
$$

Пределы интегрирования - это фактически две точки на эллиптической кривой $H(u, v)=h$. Конечно же, $\tau$ не зависит от начальной и конечной точек на кривой. Существует один замечательно простой выбор начальной точки: $u=0$, произведение $u v$ конечно, $\dot{u}=u^{\prime}=b_{1}$. После перемасштабирования $u=b_{1} x$ это дает

$$
\tau(h)=\int_{0}^{1} \frac{d x}{\sqrt{\left(1-h x+b x^{2}\right)^{2}+4 x(1-x)(a-c x)}} .
$$

Определим “действие"

$$
\mathcal{A}(h)=\int^{h} d \chi \tau(\chi) .
$$

Очевидно, определяя эту величину как “действие", мы полагаем $\int d u / \dot{u}$ "углом" в смысле переменных действие-угол. Нижний предел в (26) несушествен. Интегрирование по $\chi$ может быть выполнено, в результате получим

$$
\begin{aligned}
\mathcal{A} & =\mathcal{A}(a, b, c ; h)=\mathcal{A}(b, a, c ; h)= \\
& =-\int_{0}^{1} \frac{d x}{x} \ln \left(\frac{1-h x+b x^{2}+\sqrt{\left(1-h x+b x^{2}\right)^{2}+4 x(1-x)(a-c x)}}{2}\right) .
\end{aligned}
$$

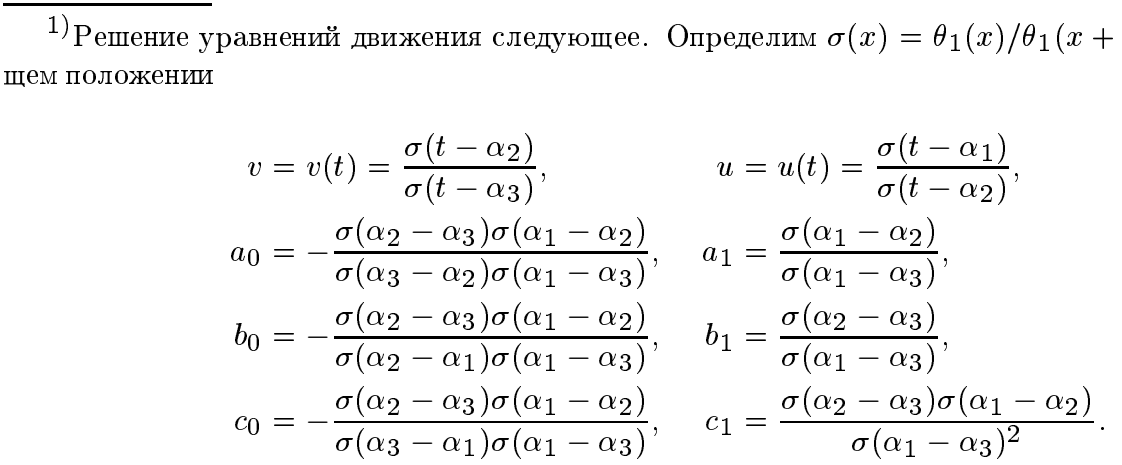

Переменная $t$ здесь пропорциональна переменной, используемой в (22). Периодичность решения объясняет термин "маятник". Сдвиг по времени $\tau$ пропорционален $\alpha_{1}-\alpha_{3}+\eta$. 
Чтобы установить симметрии $a \leftrightarrow b$, удобно репараметризовать кривую $H(u, v)=h$ следуюшим образом: пусть

$$
u=b_{1} x, \quad v=-\frac{(1-x)}{x\left(a_{0}-c_{0} b_{1} x\right)} y^{-1},
$$

тогда

$$
h=x^{-1}+y^{-1}-x^{-1} y^{-1}+b x+a y-c x y .
$$

Формула (27) может быть переписана в очевидно симметричном виде:

$$
\mathcal{A}(h)=\frac{\pi^{2}}{6}+\int_{\text {in }}^{\text {out }} \ln y d \ln x,
$$

где in $=(x=0, y=1)$, out $=(x=1, y=0)$, а $x$ и $y$ связаны соотношением (29). Дополнительная константа

$$
\frac{\pi^{2}}{6}=-\int_{0}^{1} \frac{d x}{x} \ln (1-x) .
$$

Заметим, что интегралы вида (30) уже появлялись в работе [4].

Квантование. Вернемся теперь к квантовому случаю алгебры Вейля (2). Рассмотрим формальное представление (2) в виде

$$
\mathbf{u}|x\rangle=|x\rangle x b_{1}, \quad \mathbf{v}|x\rangle=|q x\rangle \frac{-1}{x\left(a_{0}-c_{0} b_{1} x\right)} .
$$

В этом случае можно проверить, что

$$
\mathbf{u}|0\rangle=0, \quad \mathbf{v u}|0\rangle=|0\rangle\left(-\frac{b_{1}}{a_{0}}\right),
$$

равно как с помощью точного вида (8) получим

$$
\mathbf{u}^{\prime}|0\rangle=|0\rangle b_{1} .
$$

Эти соотношения аналогичны формулам (28). Поскольку $\mathbf{u}^{\prime}=\mathbf{s u s}^{-1}$, уравнение (33) означает $\mathbf{s}^{-1}|0\rangle=|1\rangle$. Если $\left\langle\psi_{h}\right|$ определено посредством $\left\langle\psi_{h}\right| \mathbf{s}=s(a, b, c ; h)\left\langle\psi_{h}\right|$, тогда

$$
s(a, b, c ; h)=\frac{\left\langle\psi_{h} \mid 0\right\rangle}{\left\langle\psi_{h} \mid 1\right\rangle} .
$$

Это выражение для $s$ через $\left\langle\psi_{h} \mid x\right\rangle$ совпадает с (20), поскольку "уравнение Шредингера" для $\left\langle\psi_{h} \mid x\right\rangle \equiv \psi_{h}(x)$ есть

$$
\left\langle\psi_{h}|(\mathbf{h}-h)| x\right\rangle=\psi_{h}(x)\left(1-h+b x^{2}\right)-\psi_{h}(q x)+\psi_{h}\left(q^{-1} x\right) q^{-1} x(1-x)\left(a-c q^{-1} x\right)=0 .
$$


Матрица (15) возникает, если переписать (34) как одношаговую рекурсию. С другой стороны, формула (20) является подходящей дискретизацией формулы (27). В частности, таким образом может быть установлена асимптотика

$$
s(a, b, c ; h ; 1)=\exp \left\{-\epsilon^{-1} \mathcal{A}(a, b, c ; h)+O\left(\epsilon^{0}\right)\right\}
$$

при $q=e^{-\epsilon}, \epsilon \rightarrow 0$.

Наш способ "вывести" $\mathbf{s}=s(\mathbf{h})$ в терминах волновой функции, конечно же, совершенно формален. Рассмотрения становятся обоснованными, если рассматривать пару уравнений Шредингера в рамках модулярного дубля (см. [5]). Из наших рассуждений следует, что модулярно дуализованный оператор $\mathbf{s}$, определенный как произведение пяти некомпактных дилогарифмов аналогично (5), равен некомпактной версии функции $s_{q, \tilde{q}}(a, b, c ; h)$, которая есть отношение двух функций вида (18), первая из которых определена для параметра $q=e^{2 \pi i \tau}$, а вторая - для модулярно дуализованного параметра $\tilde{q}=e^{-2 \pi i / \tau}$.

Благодарности. Работа была частично поддержана грантами INTAS OPEN № 00-00055, RFBR № 01-01-00201, CGP CRDF № RM1-2334-MO-02.

\section{Список литературы}

[1] L. D. Faddeev, R. M. Kashaev. Mod. Phys. Lett. A. 1994. V. 9. P. 427-434.

[2] S. Sergeev. J. Phys. A. 1999. V. 32. P. 5693-5714.

[3] V. V. Bazhanov, N. Yu. Reshetikhin. J. Phys. A. 1995. V. 28. P. 2217-2226.

[4] S. Sergeev. An evidence for a phase transition in three dimensional lattice models. MPI Preprint № 139. Bonn: MPI, 2002.

[5] L. D. Faddeev. Lett. Math. Phys. 1995. V. 34. P. 249-254; Modular double of quantum group. In: Conférence Moshé Flato 1999: Quantization, Deformation, and Symmetries. V. 1 (Dijon, France, September 5-8, 1999). Math. Phys. Stud. V.21. Eds. G. Dito et al. Dordrecht: Kluwer, 2000. P. 149-156. 